\title{
Laser beam profile analysis of a hole coupled free-electron laser
}

\author{
R. Prazeres, ${ }^{1, *}$ J.-P. Berthet, ${ }^{1}$ F. Glotin, ${ }^{1}$ J.-M. Ortega, ${ }^{1}$ B. Biswas, ${ }^{2}$ and U. Kale ${ }^{2}$ \\ ${ }^{1}$ LCP/CLIO, Bâtiment 201P2, Université de Paris-Sud, 91405 Orsay Cedex, France \\ ${ }^{2}$ BP\&FEL Laboratory, RRCAT, Indore, India \\ (Received 16 April 2010; published 24 September 2010)
}

\begin{abstract}
We present measurements and numerical simulations of the laser beam transverse profile of the CLIO infrared free-electron laser (FEL). This FEL uses a hole coupling extraction system, which introduces important perturbation on the intracavity optical mode. We show that this intracavity mode tends to avoid the extraction hole and that the output power optimization leads to an off-axis laser alignment inside the FEL cavity.
\end{abstract}

DOI: 10.1103/PhysRevSTAB.13.090702

PACS numbers: 41.60.Cr, 42.55.-f, 42.60.- -

\section{INTRODUCTION}

Infrared free-electron lasers (FELs) are used worldwide as user facilities $[1,2]$. They have large tuning ranges, such as the CLIO facility covering from 3 to $150 \mu \mathrm{m}$ [3]. Because of the lack of wideband multilayer transmitting mirrors or beam splitters, the only practical solution is to use a hole in one mirror in order to extract the laser power. For example, at CLIO a single hole of $2 \mathrm{~mm}$ diameter leads to a reasonable extracted power between 3 and $80 \mu \mathrm{m}$. However, the intracavity optical mode varies with the laser wavelength [4] and this influences the profile of the extracted light. In addition, the machine fine-tuning is made so as to optimize the laser output power and this procedure tends to select intracavity modes that are not necessarily centered on the hole. In particular, at short wavelengths where the transverse size of the mode is the smallest, the output profile is strongly affected and does not keep the cylindrical symmetry of the system. We analyze these effects in this article.

\section{A. Optical cavity and configuration of CLIO}

The optical cavity of the "CLIO" FEL is displayed on Fig. 1. The distance between cavity mirrors is $4.8 \mathrm{~m}$. In order to allow large wavelength operation of the laser $(\lambda>50 \mu \mathrm{m})$, the undulator vacuum chamber is machined such as to have a good reflectivity and to behave as a waveguide. It is realized by using extruded aluminum which possesses a good conductivity and reflectivity. The vertical inner size of the undulator's vacuum chamber is limited to $14 \mathrm{~mm}$, in order to get undulator gap parameter " $\mathrm{k}$ " up to 2 and allow broadband wavelength tuning. The horizontal size is $35 \mathrm{~mm}$.

At a short wavelength $(\lambda<10 \mu \mathrm{m})$, the waveguide does not modify the laser mode, because it is sufficiently larger than its typical dimensions, and does not play a significant role in what follows.

\footnotetext{
*rui.prazeres@u-psud.fr
}

The output coupling is performed here by a hole of $2 \mathrm{~mm}$ in the center of downstream cavity mirror. This solution is advantageous compared to other solutions since it is wideband. However, the output coupling factor $T_{x}$ of the hole, i.e., ratio between output power and intracavity power, is strongly dependent on the transverse distribution of the laser mode at the output mirror.

\section{B. The numerical code MODES}

The numerical code we have called MODES [5,6] has been used here. It calculates the amplitude distribution $A(x, y)$ of the laser mode at saturation, in any point of the cavity, and all related parameters: the cavity losses $L$, the extraction rate $T_{x}$ of hole coupling, the intracavity laser power $P_{\text {in }}$, and the output power $P_{\text {out }}$. The cavity losses are equal to $L=\Delta P_{\text {in }} / P_{\text {in }}$, where $\Delta P_{\text {in }}$ is the power loss of the intracavity laser pulse after one cavity round trip. The extraction rate $T_{x}=P_{\text {out }} / P_{\text {in }}$ is the ratio between output

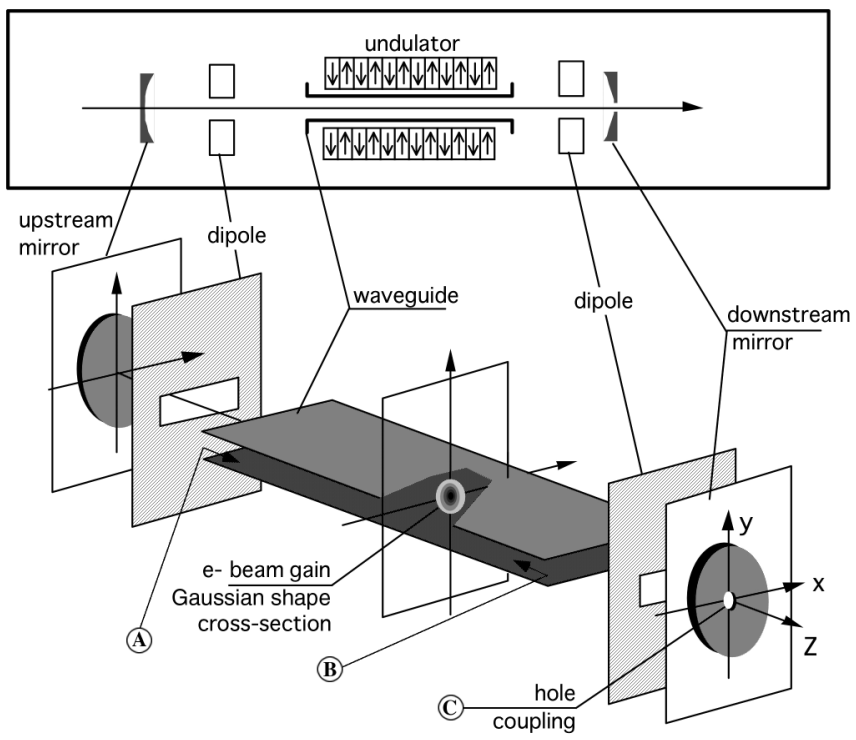

FIG. 1. Schematic layout of the CLIO free-electron laser optical cavity. 
power $P_{\text {out }}$ and intracavity power $P_{\text {in }}$. The calculation of all parameters - cavity losses, extraction rate, and intensity at saturation-gives the FEL extracted power $P_{\text {out }}[6]$. The code takes into account the configuration of the optical cavity: free-propagation areas, waveguide propagation in undulator section, mirror reflections, hole coupling, and transverse distribution of gain profile. It uses an iterative process of wave propagation $A(x, y)$ in the cavity. Starting from a Gaussian profile in undulator $A_{0}(x, y)$, it converges to a steady state mode profile $A(x, y)$, which corresponds to the saturation regime of the FEL. The convergence is reached when the losses coefficient $L$ becomes stable within $10^{-3}$ of relative variation for successive cycles. It requires generally about 50 to 200 cycles to get convergence.

The calculation of the power at laser saturation is based on the theory of FEL intensity saturation [7]. The exponential gain takes into account the nonlinear behavior of small signal gain in the high gain regime; it includes the inhomogeneous broadening of the filling factor and the longitudinal mode coupling factor. It is a single wavelength theory, which does not include short pulse effects of the FEL and is limited to narrow linewidth FEL operation.

\section{MEASUREMENTS}

The optimization of the FEL corresponds to a compromise between hole coupling extraction and intracavity laser power. Indeed, the "natural" tendency of the intracavity laser mode is to reduce the optical losses, i.e., to create a mode with a minimum of hole coupling, leading to a maximum intracavity power, whereas the desire of the FEL user is to get a maximum of output power, which is proportional to the hole coupling. Therefore, the machine parameters are adjusted so as to obtain the maximum FEL extracted power. This compromise is mainly dependent on the relative values of two parameters: the laser wavelength and the extraction hole size. For "small" hole size, or long wavelength, the laser profile is not strongly affected. But for "large" hole size the laser profile tends to exhibit, on the output mirror, a minimum on the $Z$ axis which corresponds to a minimum of hole coupling extraction rate. We define here the $Z$ axis by two points which are the centers of both cavity mirrors (one of them being the extraction hole). Nevertheless, the better size of hole coupling (larger output FEL power), produces a distorted transverse profile of the output laser beam.
In order to have a diagnostic of this effect, it would be interesting to have an image of the intracavity laser mode. Because of the physical configuration of CLIO, this is impossible, therefore we have done measurements of the laser profile outside of the cavity.

Measurements of the laser beam profile have been done on CLIO with a $2 \mathrm{D}$ infrared camera. The profiles have been measured outside of the optical cavity. The experimental setup is displayed in Fig. 1: a focusing mirror is installed at $1.65 \mathrm{~m}$ from the laser output, and the measurement is carried out in the experimental room at about $14 \mathrm{~m}$ distance. In order to fit the $2 \mathrm{~cm}^{2}$ area of the detector, the optical beam is focused again with a spherical mirror in an experimental room. The detector is a $2 \mathrm{D}$ matrix of pyroelectric detectors. The image, which is projected on the surface of detector, corresponds to the magnified image of the hole on output downstream mirror M2. The astigmatism due to slightly "off-axis" illumination of spherical mirrors is negligible ( $\pm 0.4 \%$ of variation of focal length on both focal planes).

The measured profile is shown, for different wavelengths, in Fig. 3. At short wavelength $(\lambda<10 \mu \mathrm{m})$, as displayed in Fig. 3(a) for $\lambda=6.5 \mu \mathrm{m}$, the profile always exhibits a typical "crescent moon" shape. Even when trying to modify all parameters of the FEL, we always obtain this typical profile. This shows that the intracavity laser mode is not centered on the $Z$ axis, and only one offaxis part of the profile is extracted by the hole. As the wavelength is increased, as shown in Fig. 3(b) for $\lambda=$ $17 \mu \mathrm{m}$, the profile tends to fill the hole, as expected. The horizontal lines are due to diffraction in the laser beam line- outside of the cavity. Indeed, the large laser intensity profile produced by the first lens (Fig. 2) tends to be diffracted in the $14 \mathrm{~m}$ long beam line by the edge of the mirrors.

\section{SIMULATIONS}

For a given laser wavelength and with a given hole coupling size, there are two FEL parameters which may be adjusted by users in order to optimize the laser output power: (1) the tuning of angular tilt of cavity mirrors, and (2) the electron beam alignment in the undulator section. These parameters are crucial for the output laser power and have an influence on the intracavity laser mode. The compromise which is introduced above, between hole extraction and intracavity laser power, is reached in practice by

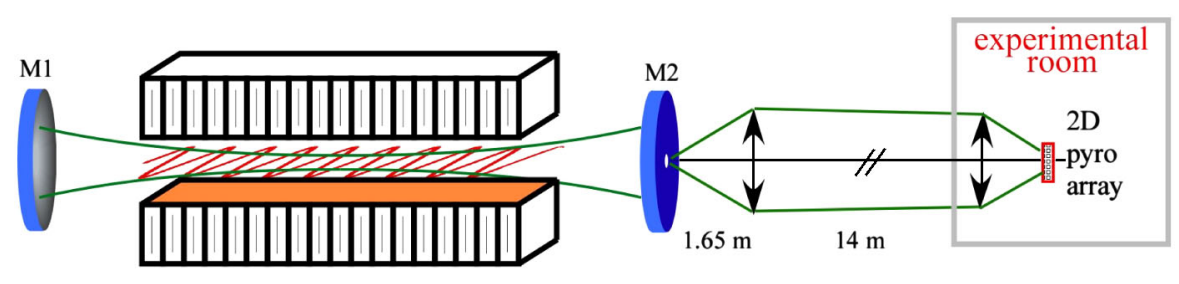

FIG. 2. Experimental setup of the laser profile measurement. 

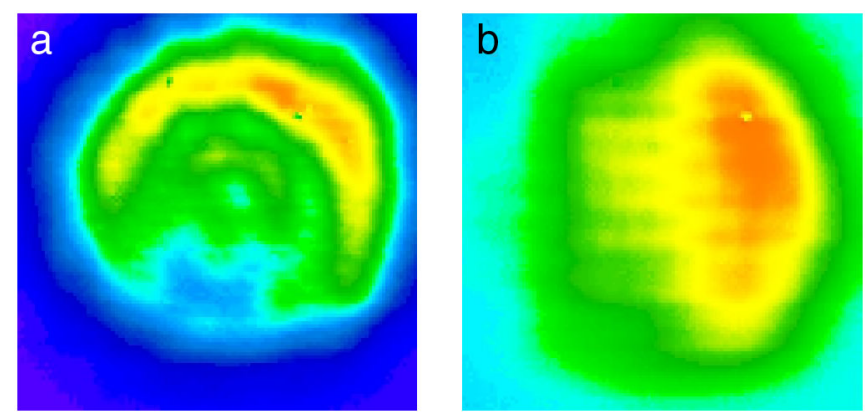

FIG. 3. Measurement of laser mode transverse profiles, in the experimental room, at $\lambda=6.7 \mu \mathrm{m}$ (a), and $\lambda=17 \mu \mathrm{m}$ (b).

using a slight misalignment of the optical cavity. This breaks the axial symmetry of the laser mode and allows one to optimize the hole coupling extraction rate. Indeed, the numerical simulations show that the output power may be increased by using a tilt $\theta$ on the output downstream mirror (while keeping the upstream mirror at normal incidence). Figure 4 shows an example of output power as a function of mirror tilt $\theta$, for $\lambda=6.5 \mu \mathrm{m}$, corresponding to the measurements of Fig. 3(a). A maximum of output power is obtained close to $\theta=0.5 \mathrm{mrad}$. A change of the optical mode takes place at this value, which explains the sharp edge on the curve of extracted power. Indeed, there is a competition between both mode patterns, shown in Fig. 6(a) for $0.56 \mathrm{mrad}$ and Fig. 8(a) for $0.4 \mathrm{mrad}$. The final solution does not make a compromise (a linear combination) between these two modes, and it has to "choose" one of them. This is the reason of the discontinuity of curve in Fig. 4.

In practice, the mirror is not tilted by such an amount that would result in the loss of the cavity prealignment. Instead, we tilt the electron beam by an equivalent amount in the horizontal plane, where the (vertical) magnetic field is sufficiently homogeneous within a few millimeters. However, the beam position measurement is not sufficiently accurate in order to measure its angle with respect to the mechanical and optical axis.

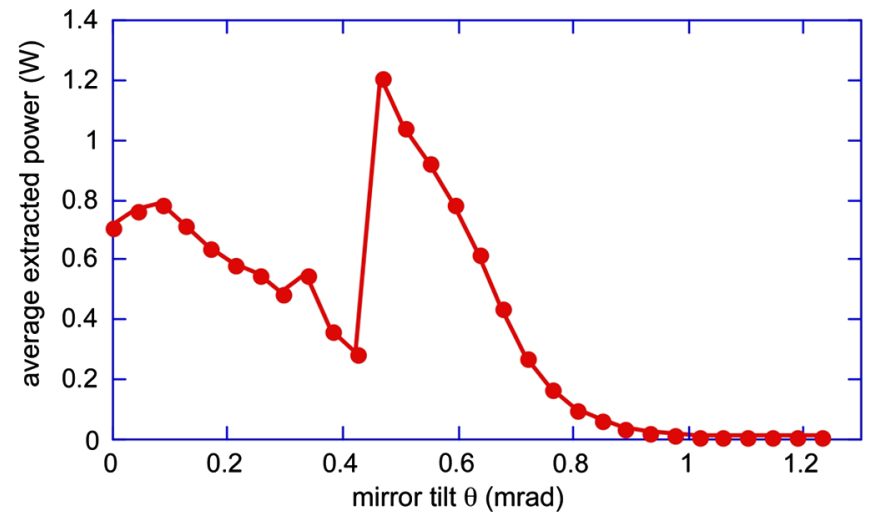

FIG. 4. Numerical simulation of output laser power versus a tilt of the output mirror.
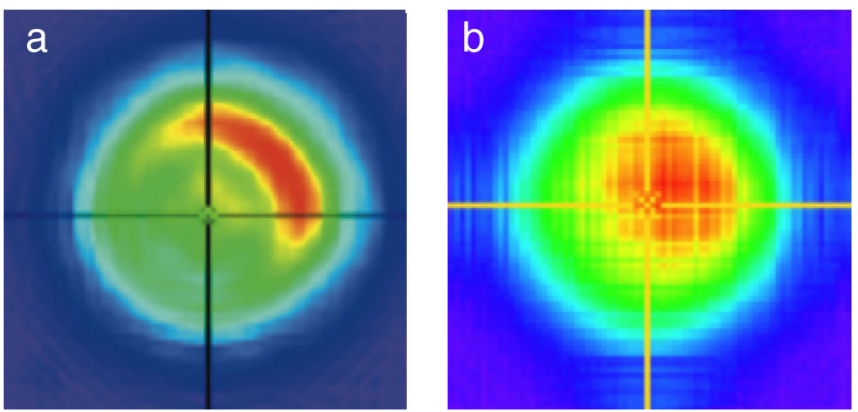

FIG. 5. Numerical simulation of the laser mode transverse profile, calculated in the experimental room, on the detector plane, at $\lambda=6.7 \mu \mathrm{m}$ (a), and $\lambda=17 \mu \mathrm{m}$ (b).

Figure 5 displays a numerical simulation of the amplitude distribution $A(x, y)$, calculated in the experimental room. It takes into account the experimental conditions of measurements: it calculates the wave propagation along the optical beam line and through the focusing element up to the detector plane represented in Fig. 2. The wavelengths are respectively $\lambda=6.5 \mu \mathrm{m}$ (a) and at $\lambda=17 \mu \mathrm{m}$ (b). These profiles have been calculated using a tilt on output downstream mirror M2. This tilt is oriented of $45^{\circ}$, with $\theta_{x}=\theta_{y}=0.4 \mathrm{mrad}$, in order to get $\theta=0.56 \mathrm{mrad}$ at $45^{\circ}$ in the $X Y$ plane. These images may be compared to the image on Fig. 3, which have been measured in the same conditions with the pyroelectric camera. The profile on Fig. 5(a) exhibits a crescent moon shape which reproduces reasonably well the measurement. The profile on Fig. 5(b) is larger and fills the hole size, as shown in measurements on Fig. 3(b).

Figure 6 shows a simulation within the same conditions, but it displays the profile inside of the cavity, on the output downstream mirror, at $\lambda=6.7 \mu \mathrm{m}$ (a) and $\lambda=17 \mu \mathrm{m}$ (b). The diameter of mirror $(38 \mathrm{~mm})$ is represented by a circle, and the extraction hole $(2 \mathrm{~mm})$ by a black dot in the center. The laser profile exhibits a single spot which is off axis at $45^{\circ}$ orientation, like the downstream mirror. At $17 \mu \mathrm{m}$, the position of the hole is closer to the center of the laser distribution. This is sufficient to create a rather homogeneous laser profile on the experimental room [Fig. 5(b)].
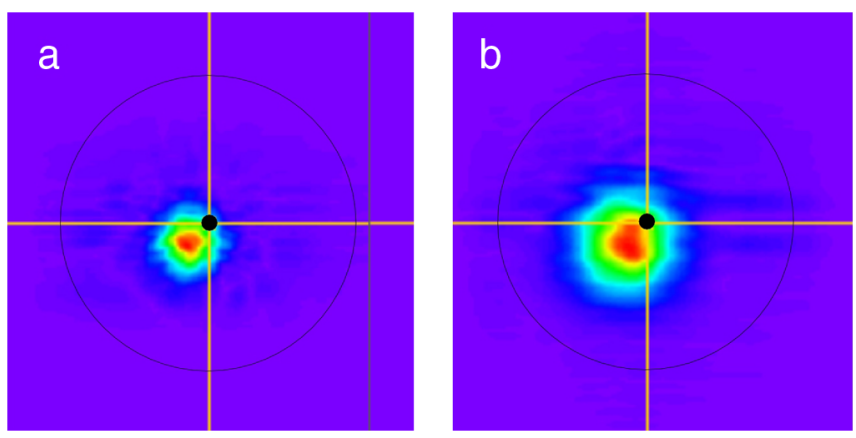

FIG. 6. Numerical simulation of the laser mode profile on the downstream mirror, (a) at $\lambda=6.7 \mu \mathrm{m}$, and (b) at $\lambda=17 \mu \mathrm{m}$. 

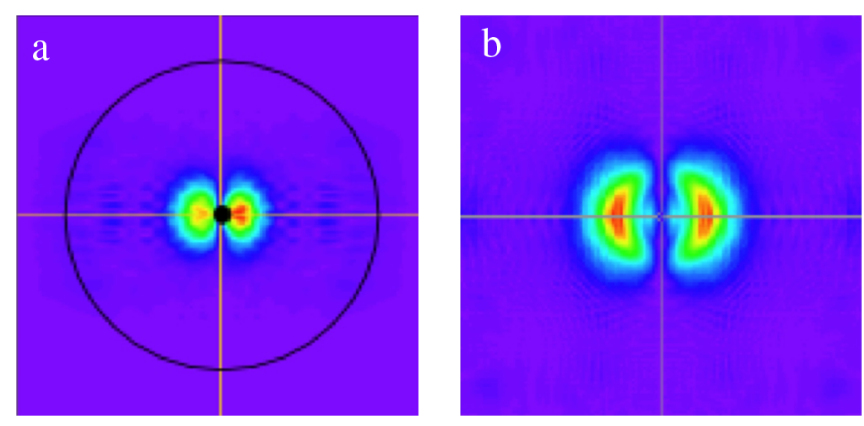

FIG. 7. Numerical simulation of the laser mode profile, for $\lambda=6.7 \mu \mathrm{m}$, at normal incidence at the downstream mirror, (a) on cavity output mirror, and (b) in experimental room.

In real conditions, during FEL adjustment, the optimum tilt for the output mirror uses a combination of both axes $X$ and $Y$, but we have no measurement of the angular tilt with respect to the optical axis. The simulation on Fig. 5 using an orientation of $45^{\circ}$ fits very well the profile measurement in Fig. 3. This shows that the intracavity laser mode "avoids the extraction hole" when optimizing the output FEL power.

In order to show a comparison with on-axis aligned cavity, Fig. 7 displays the result of the numerical simulation calculated for $\theta=0$, i.e., for normal incidence at the downstream mirror. In this case, the intracavity mode exhibits a double spot in Fig. 7(a), with a minimum on the extraction hole. In Fig. 7(b), corresponding to the profile in the experimental room, the mode profile also exhibits a double symmetrical spot. In practice, we never observed, in the experimental room, such a laser profile as it corresponds to an extremely weak extracted power. This is another argument to say that the laser mode is never aligned on the $Z$ axis ( $Z$ axis being defined by the two centers of cavity mirrors).

It is also interesting to look by simulation (Fig. 8) at the laser profile that would be obtained at the angle $\theta=0.4 \mathrm{mrad}$, corresponding to a minimum of output power in the curve of Fig. 4.

The mode is obviously orientated at $45^{\circ}$, because of the orientation of the output mirror. The profile, on output mirror in Fig. 8(a), exhibits a minimum of amplitude on the extraction hole, which explains the minimum of extracted power in Fig. 4.

Indeed there is a competition in the FEL configuration between the hole coupling and the amplification process: the laser mode is amplified, in the undulator section, within an area corresponding to the electron beam cross section. This cross-section area is small, compared to the fundamental cavity mode size. The electron beam is centered on the $Z$ axis, i.e., on an axis passing through the extraction hole and tends to force the laser mode to be aligned on the extraction hole ("automatic hole alignment"). This effect is very important to get important output laser power, as it forces the system to keep a large extraction rate $T_{x}$. In order
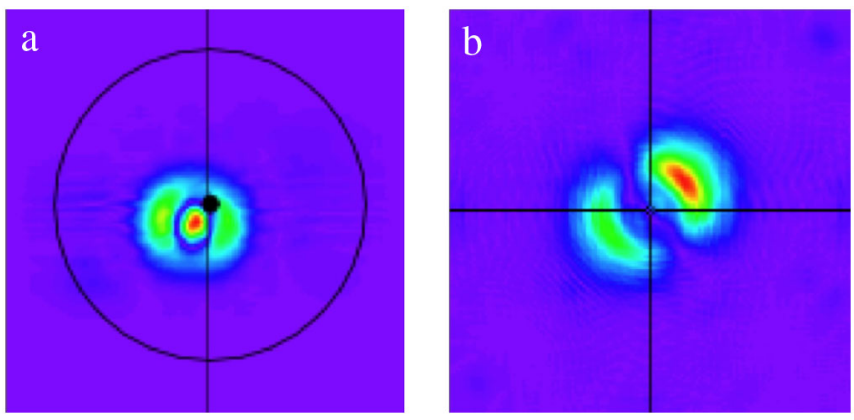

FIG. 8. Numerical simulation of the laser mode profile with a tilt $\theta=0.4 \mathrm{mrad}$ at $45^{\circ}$ on the downstream mirror, (a) on cavity output mirror, and (b) in the experimental room.

to point out this effect, a numerical simulation has been done within the same conditions as above, but using a flat electron beam profile (large cross section). This produces a uniform amplification of the steady state laser profile, and avoids the effect of automatic hole alignment. Within these (nonrealistic) conditions, the simulation gave quite zero extraction rate $T_{x}$, and the output power was zero for any angular tilt of the downstream mirror. This confirms the importance of the laser focusing effect, induced by the small electron beam cross section, upon the extraction hole.

\section{CONCLUSIONS}

We have studied the optical mode output profile provided by hole coupling, with the CLIO infrared freeelectron laser. At the shortest wavelength of the tuning range, we always observed that this laser profile contains distorted structures. The measurements we have done, using a 2D camera, and the numerical simulations confirm the observation. This effect is due to the perturbation created by the hole coupling extraction system of the FEL cavity. In practice, for the CLIO free-electron laser, the $2 \mathrm{~mm}$ size hole allows a FEL tuning range from $\lambda=5 \mu \mathrm{m}$ up to about $40 \mu \mathrm{m}$. A larger hole ( 3 or $4 \mathrm{~mm}$ ) is required within the range $\lambda=20 \mu \mathrm{m}$ up to about $150 \mu \mathrm{m}$. Nevertheless, there is no alternative outcoupling system working within an order of magnitude of the tuning range and creating no cavity length dependence of wavelength (such as an intracavity Brewster plate).

\section{ACKNOWLEDGMENTS}

This work has been realized with funding from the Institut de Chimie-CNRS and IFCPAR CEFIPRA Contract No. 3706-1.

[1] J. M. Ortega, Nucl. Instrum. Methods 507, 8 (2003).

[2] W. B. Colson, J. Blau, K. Cohn, J. Jimenez, and R. Pifer, Free-electron Lasers in 2009, http://cern.ch/AccelConf/ FEL2009/papers/wepc43.pdf. 
[3] J. M. Ortega, F. Glotin, and R. Prazeres, Infrared Phys. Technol. 49, 133 (2006).

[4] R. Prazeres, F. Glotin, and J.-M. Ortega, Phys. Rev. ST Accel. Beams 12, 010701 (2009).

[5] R. Prazeres and V. Serriere, Nucl. Instrum. Methods Phys. Res., Sect. A 475, 524 (2001).
[6] R. Prazeres, Eur. Phys. J. Appl. Phys. 16, 209 (2001).

[7] G. Dattoli, J. Appl. Phys. 84, 2393 (1998); G. Dattoli and P. L. Ottaviani, Opt. Commun. 204, 283 (2002); G. Dattoli, T. Letardi, J. M. J. Madey, and A. Renieri, Nucl. Instrum. Methods Phys. Res., Sect. A 237, 326 (1985). 